\title{
The Structure of Mitochondria: A Study Using High-Pressure Freezing and STEM Tomography
}

\author{
P. Walther,* L. Wang, ** and K. Höhn* \\ * Central Facility for Electron Microscopy, Ulm University, 89069 Ulm, Germany \\ ** Institute of Virology, University Hospital Ulm, 89081 Ulm, Germany
}

The structure of organells as observed in the electron microscope depends from the specimen preparation method used. It is generally believed that an intermembrane space of about 40 to 100 $\mathrm{nm}$ separates inner and outer mitochondrial membrane and the membranes of the cristae; and according to textbooks this space bears a number of different proteins for oxidative phosphorylation and for control of apoptosis [1]. This concept is derived from electron microscopical samples chemically fixed with glutharaldehyde [2, and others]. The size of the intermembrane space is described to be rather constant, although form and shape of the mitochondria and of the cristae vary between cell types and physiological states [2]. Considerably smaller intermembrane spaces, however, have been observed when cryo-fixation methods have been used [3, and others].

For this study we used high pressure freezing for fast immobilization and fixation of cultivated cells under conditions as physiological as possible. We have found, that the width of the intermembrane space depends upon the first immobilization or fixation step, among other parameters. When samples are fixed with glutaraldehyde prior to high pressure freezing (Fig. 1) a large intermembrane space occurs. When high pressure freezing, however, is performed from a physiological state, the intermembrane space in mitochondria is very small in many cells and tissue pieces investigated (Figs. 2 and 3). Fig. 2 shows a mitochondrion in a Pancl cell and Fig. 3 is a mitochondrion in a macrophage visualized by dark field scanning transmission electron tomography (STEM) [4]. In order to exclude artefact formation during freeze-substitution, we compared the results with data from yeast after HPF and cryo-fracturing in the cryo-SEM [5]. We found inner and outer membranes in close apposition in these samples too.

Our findings of a very small intermembrane gap in healthy mitochondria correspond well with older studies on cryofixed isolated mitochondria by spray freezing [6], or propane jet freezing [7]. When frozen in a metabolically active state, inner and outer membranes were in close apposition, whereas when respiration was blocked by antimycin A, the intermembrane space became large [6]. The cell biological implication of these findings is, that in a respiratory active mitochondrion all intermembrane proteins must be in close touch with the membranes.

[1] B. Alberts et al., Mol. Biol. of the Cell, 5th ed. (2008) Garland Publ., New York, NY

[2] M. G. Sun et al., Nature Cell Biol. 9 (2007) 1057-1065.

[3] D. Nicastro et al., J. Struct. Biol. 129 (2000) 48-56.

[4] K. Höhn et al., Histochem. Cell Biol. 135 (2011) 1-9.

[5] P. Walther et al. Proceedings of MC 2009, vol. 2, Verlag der TU Graz, pp 49-50.

[6] R. D. A. Lang and J. R. Bronk, J. Cell. Biol. 77 (1978) 134-147.

[7] G. Knoll and D. Brdiczka, Biochimica et Biophysica Acta 733 (1983) 102-110. 

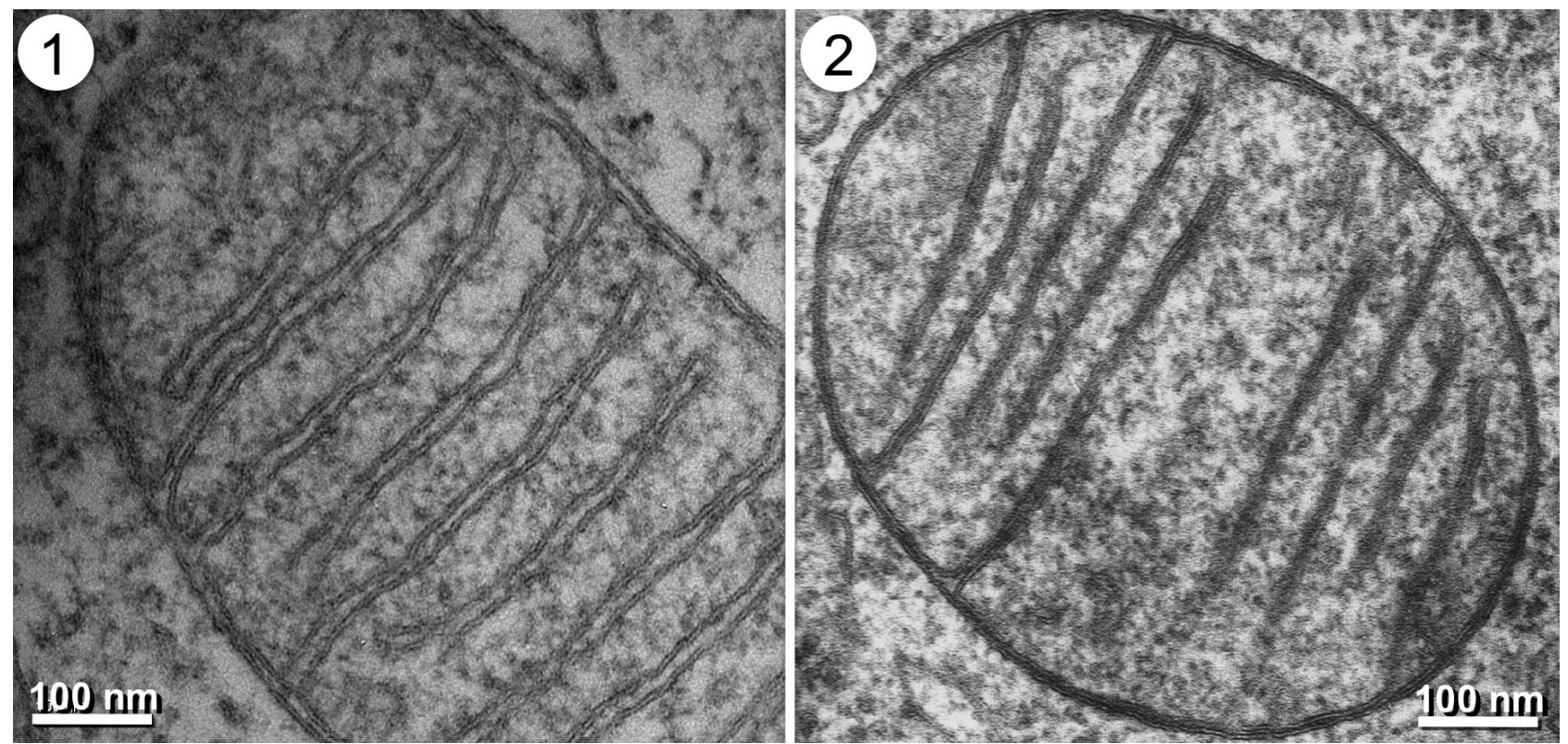

Figs. 1 and 2. Mitochondria in ultrathin sections from cultivated cells, that where either immobilized by glutaraldehyde fixation and afterwards high-pressure frozen and freeze substituted (Fig.1), or directly high pressure frozen and freeze substituted (Fig. 2). Whereas a large intermembrane space occurs in the cristae of the glutaraldehyde fixed sample (Fig.1), the membranes in the cristae of the high-pressure frozen sample are in close apposition (Fig. 2).
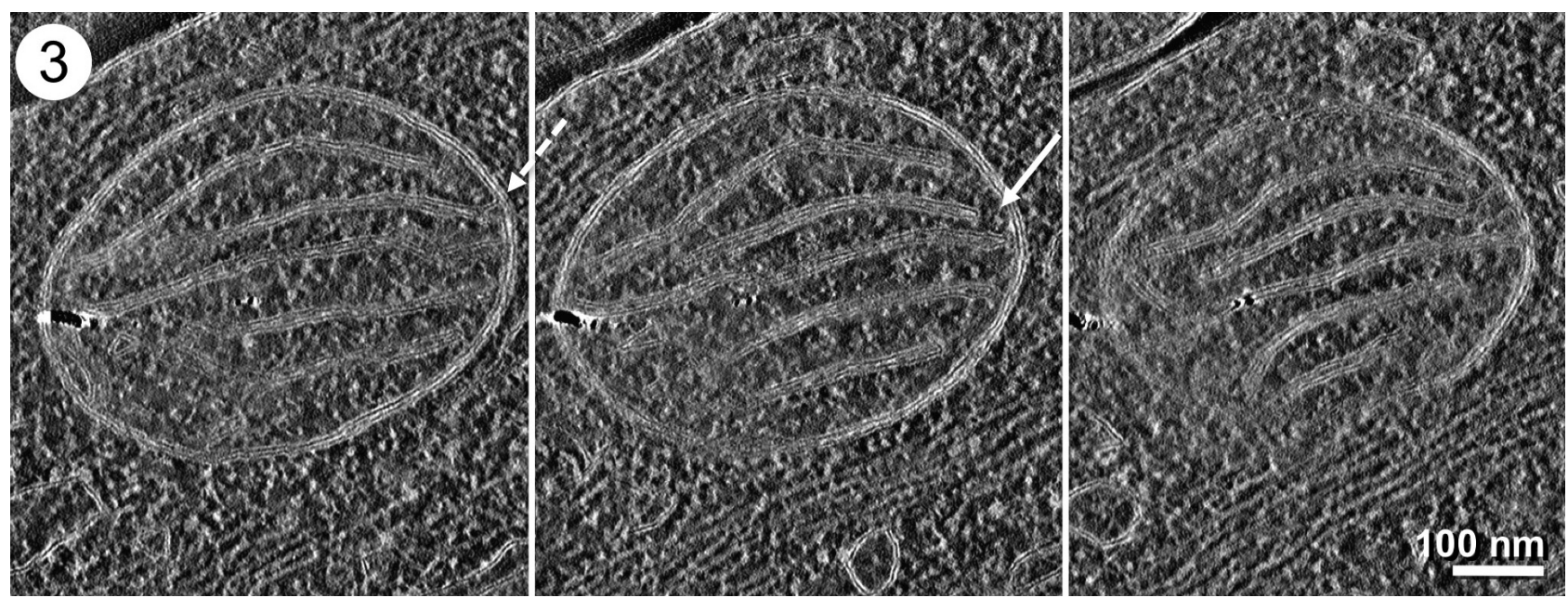

Fig. 3. Dark field scanning transmission electron (STEM) tomography of a mitochondrion in a human macrophage. Three reconstructed virtual sections are shown. The sample was tilted from +72 to $-72^{\circ}$ for recording the tomogram at $300 \mathrm{kV}$ accelerating voltage. Inner and outer mitochondrial membrane as well as the membranes in the cristae are in close apposition, no intermembrane space is visible. The dashed arrow depicts a cristae membrane that forms a continuity with the inner membrane, whereas the arrow in the next image depicts a gap between the same crista and the inner membrane. This observation is in agreement with the cristae junction model. 